\title{
ON 3-MANIFOLDS THAT ARE NOT SIMPLY CONNECTED
}

\section{KYUNG WHAN KWUN}

Let $X$ be a connected 3-manifold. Then $X$ is simply connected if and only if each simple closed curve in $X$ lies in a simply connected neighborhood. On the other hand, each point of $X$ trivially lies in a simply connected neighborhood with or without $X$ itself simply connected. However, we will show that arcs are big enough to reflect the global property of $X$ as to simple connectedness in the sense that $X$ is simply connected if and only if each arc in $X$ lies in a simply connected neighborhood.

Theorem. Let $X$ be a 3-manifold that is not simply connected. Then there exists an arc in $X$ that does not lie in any simply connected neighborhood.

Proof. There exists a simple closed polygon $L$ that is not deformable to a point over $X$, i.e., the inclusion map of $L$ into $X$ is essential. To see this, we choose a loop representing a nontrivial element of the fundamental group of $X$ and apply thereto the simplicial approximation theorem followed by obvious modifications if necessary. Let $T$ be a polyhedral solid torus which is a closed tubular neighborhood of $L$. Evidently, $T$ does not lie in a simply connected neighborhood.

We now proceed to construct a Cantor set of Antoine type [1]. Let $T_{1}, T_{2}, \cdots, T_{k}$ be a finite number of polyhedral solid tori of diameter less than $1 / 2$ that are situated in Int $T$ as suggested by Figure 1 . We show that the sum $T^{1}$ of the $T_{i}$ does not lie in a simply connected neighborhood. Suppose it does.

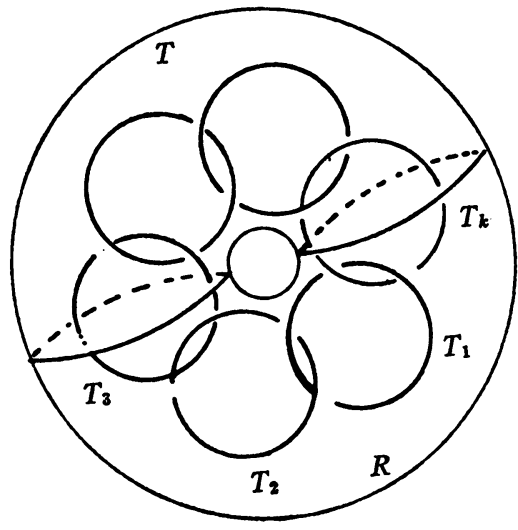

FIGURE 1

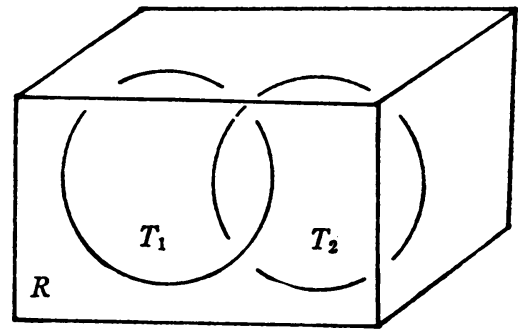

FIGURE 2

Received by the editors March 16, 1961. 
Let $U$ be a simply connected open set containing $T^{1}$. We cut a polyhedral cube $R$ containing $T_{1}$ and $T_{2}$ out of $T$ as suggested by Figures 1 and 2. $T_{1}$ and $T_{2}$ are linked in $R$ in the sense that neither of the two can be deformed to a point over the complement in $R$ of the other. We show that they can be joined by an arc in Int $R \cdot U$. Let $L_{i}$ be simple closed polygon that circle on $\mathrm{Bd} T_{i}$ longitudinally once and $x_{i}$ be points on $L_{i}, i=1,2$. Since $U$ is simply connected, there exists a singular disk $D$ bounded by $L_{1}, D \subset U$. We may suppose that $D$ meets $\mathrm{Bd} R$ at a 1-dimensional set. Let $D^{\prime}$ be the component of $D$. Int $R$ that contains $L_{1}$. Then $D^{\prime}+\mathrm{Bd} R$ contains a singular disk $D^{\prime \prime}$ bounded by $L_{1}$. Since $D^{\prime \prime}$ meets $L_{2}$ and $L_{2}$ does not meet $\operatorname{Bd} R$, the existence of the required arc is established.

Let $C_{1}$ be an arc in Int $R \cdot U$ that joins $x_{1}$ and $x_{2}$. Similarly, we construct $\operatorname{arcs} C_{i}$ joining $x_{i}$ and $x_{i+1}, x_{i} \in T_{i}$, subscripts being taken modulo $k$, such that $\sum C_{i}$ is a simple closed curve in $U$ which is deformable to $L$ in Int $T$. Since $U$ is simply connected, $L$ is deformable to a point over $X$, contradictory to the choice of $L$. Thus $T^{1}$ does not lie in a simply connected neighborhood.

We now construct polyhedral solid tori $T_{i 1}, T_{i 2}, \ldots$ in Int $T_{i}$ such that each $T_{i j}$ is of diameter less than $1 / 4, T_{i j}$ are situated in Int $T_{i}$ as $T_{j}$ are in Int $T$. Let $T^{2}$ be the sum of $T_{i j}$. Then $T^{2}$ does not lie in a simply connected neighborhood. For if it did, then the simply connected neighborhood must contain for each $i$ a simple closed polygon in Int $T_{i}$ that is deformable within $T_{i}$ to a simple closed polygon that lies on Bd $T_{i}$ and circles $T_{i}$ longitudinally once, and which in turn would imply that it contained a curve which is deformable to $L$ in $X$, thus the same contradiction.

Similarly, we construct sets $T^{3}, T^{4}, \cdots$ such that no $T^{i}$ lies in a simply connected neighborhood, each $T^{i}$ contains $T^{i+1}$ in its interior and the intersection of $T^{i}$ is a Cantor set $C$. There exists in Int $T$ an arc $P$ containing $C$. Then $P$ does not lie in any simply connected neighborhood in $X$.

We wish to thank the referee for his suggestion which made the proof considerably shorter.

\section{REFERENCE}

1. L. Antoine, Sur l'homeomorphie de deux figures et de leur voisinapes, J. Math. Pures Appl. 86 (1921), 221-325.

The Seoul National University, Seoul, Korea 